\title{
Fabrication of Chitosan Nanofiltration Membrane by Film Casting Strategy for Effective Removal of Dye/Salt in Textile Wastewater
}

Qingwu Longa,b,e Zhe Zhang c, Guangxian Qid ${ }^{\mathrm{d}}$, Zhu Wanga ${ }^{\mathrm{a}}$, Yibo Chen ${ }^{\mathrm{a}}$, Zhao-Qing Liu ${ }^{\mathrm{a}, *}$

aSchool of Chemistry and Chemical Engineering/Institute of Clean Energy and Materials/Guangzhou Key Laboratory for Clean Energy and Materials, Guangzhou University, Guangzhou 510006, PR China.

${ }^{b}$ College of Chemistry and Chemical Engineering, Qiannan Normal University for Nationalities, Duyun 558000, China

'Institute of Environmental Research at Greater Bay/Key Laboratory for Water Quality and Conservation of the Pearl River Delta, Ministry of Education, Guangzhou University, Guangzhou 510006, China

${ }^{\mathrm{d}}$ Guangdong Key Laboratory of Membrane Materials and Membrane Separation/Guangzhou Institute of Advanced Technology, Chinese Academy of Sciences, Guangzhou 511458, China

eGuangdong Provincial Key Laboratory of Petrochemical Pollution Process and Control, Guangdong University of Petrochemical Technology, Maoming, 525000, China

* E-mail addresses for the Corresponding author: 1zqgzu@gzhu.edu.cn (Z.-Q. Liu)

Number of pages: 10 (S1-S10)

Number of figures: 9 (Figure S1-S9) 


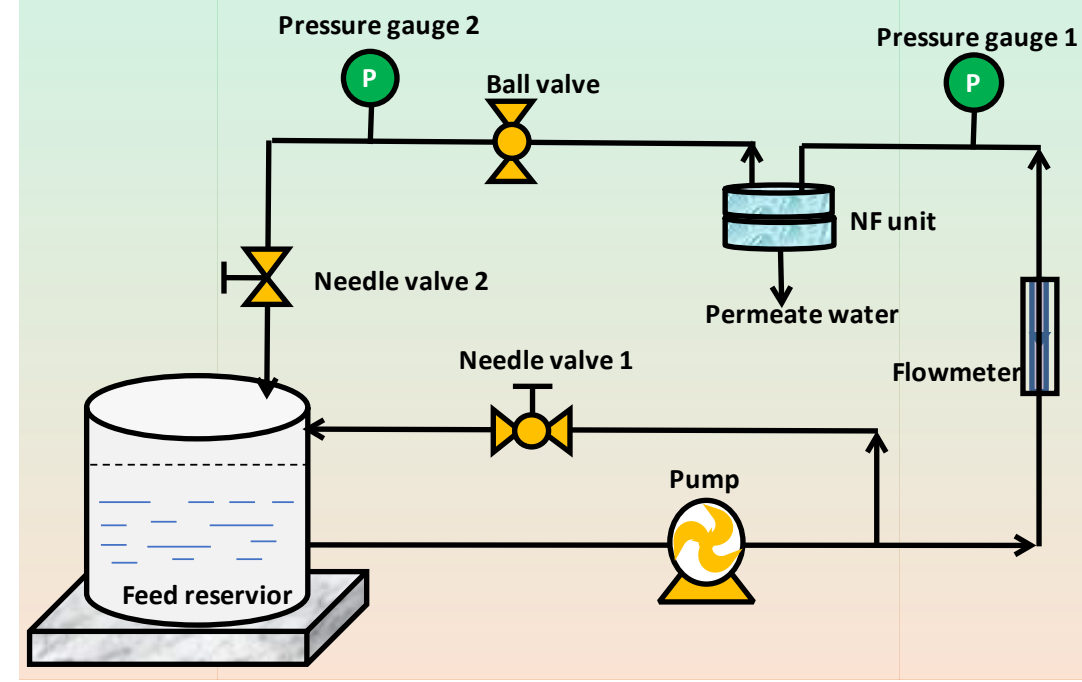

Figure S1. The NF apparatus for evaluating the performance of CS membranes. 


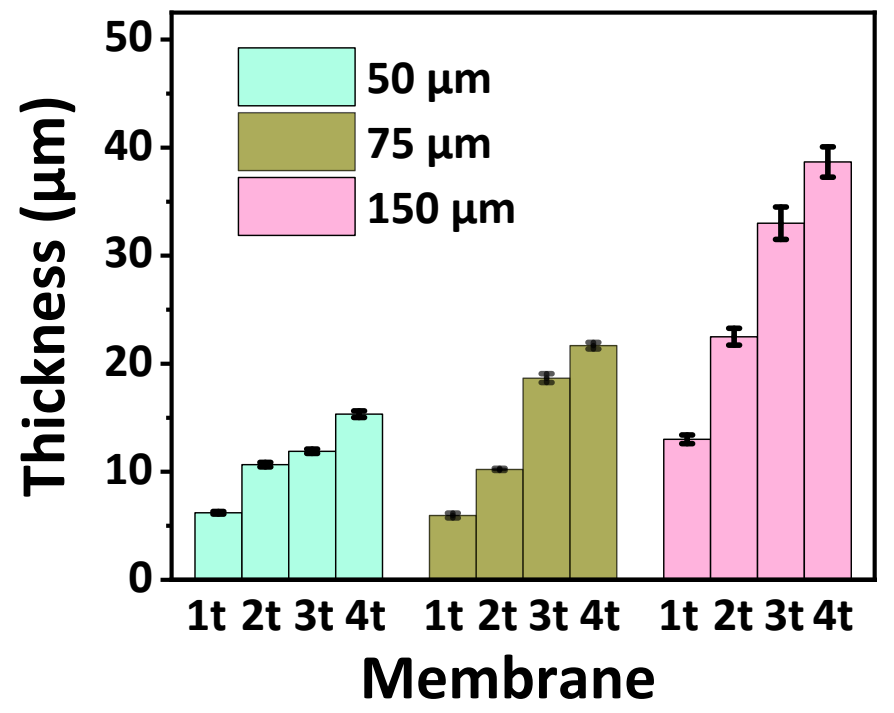

Figure S2. The thickness of series of CS membranes. 


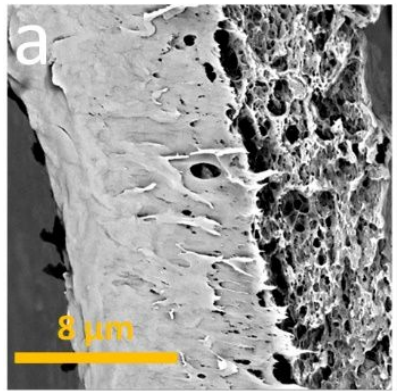

75-1t

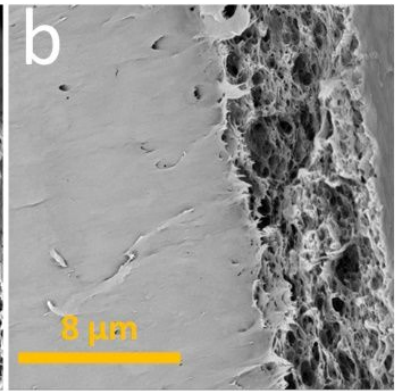

75-2t

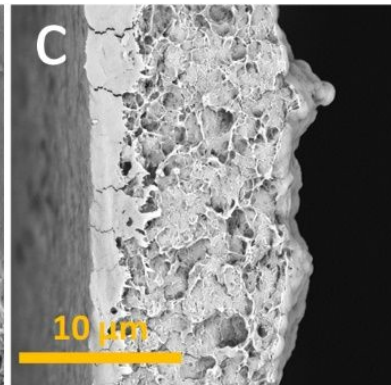

75-3t

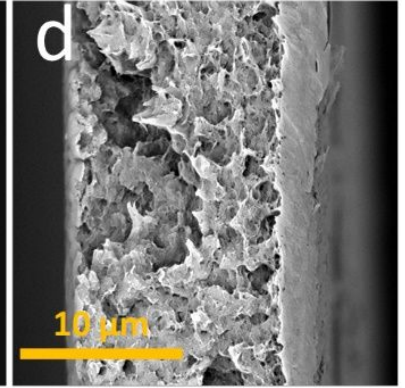

$75-4 t$

Figure S3. Cross-sectional SEM images of the membrane 75-nt at the magnification of 10,000 times. (a) membrane 75-1t, (b) membrane 75-2t, (c) membrane 75-3t and (d) membrane $75-4 \mathrm{t}$ 

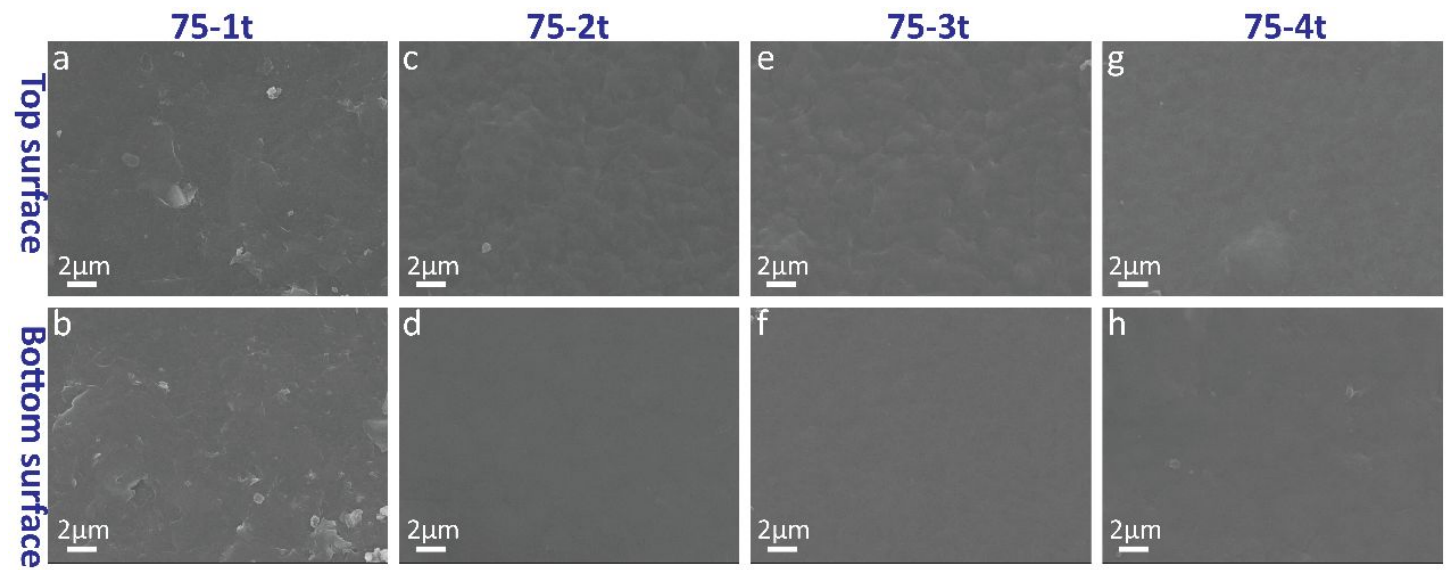

Figure S4. Top (up) and bottom (down) surface morphologies of the membrane 75-nt at the magnification of 15,000 times. The top surface of membrane 75-1t (a), 75-2t (c), 75-3t (e), and 75-4t; $(\mathrm{g})$ the bottom surface of membrane 75-1t (b), 75-2t (d), 75-3t (f) and 75-4t (h). 


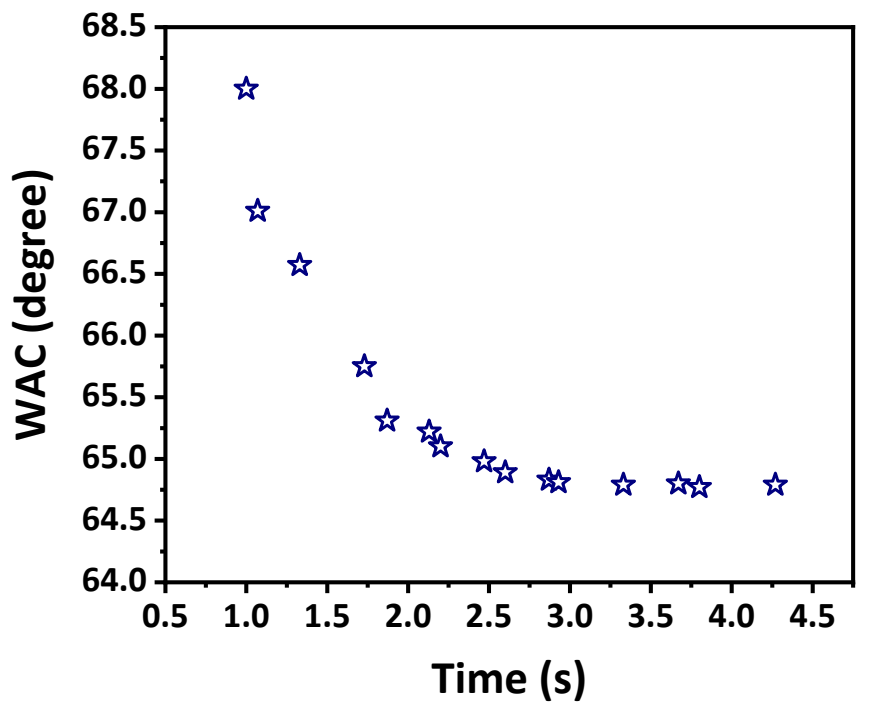

Figure S5. The dynamical water contact angles of membrane $75-4 \mathrm{t}$ 


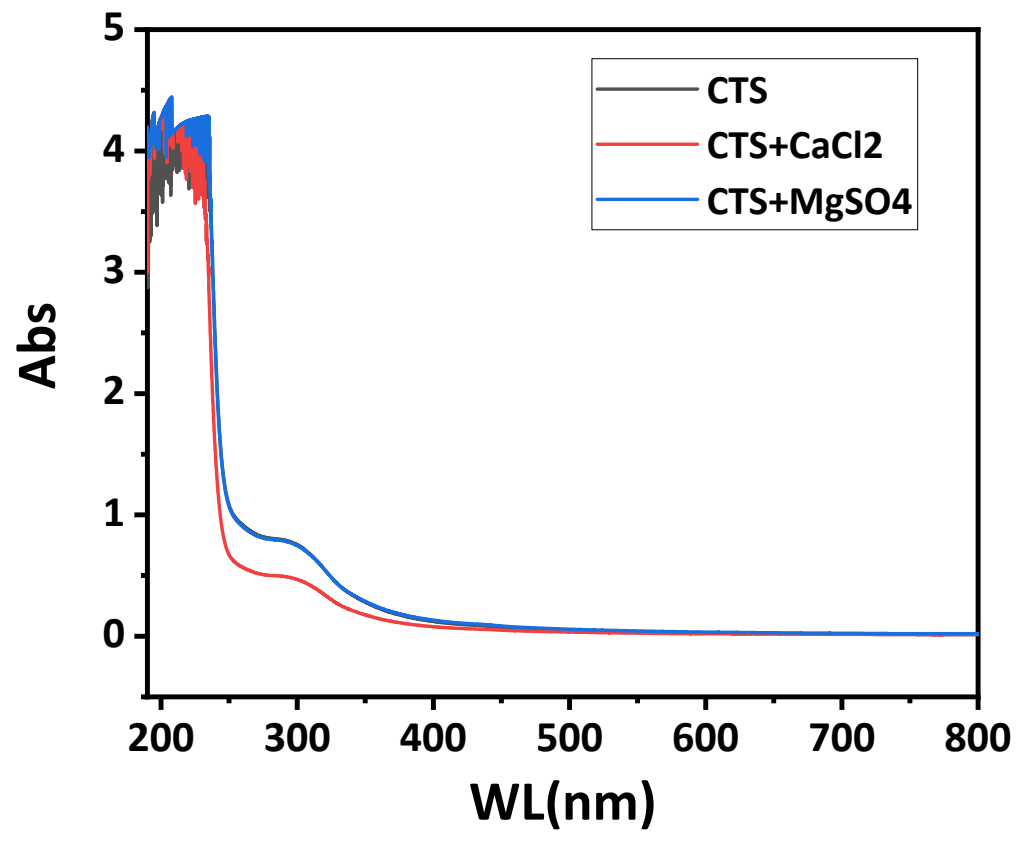

Figure S6. The UV-Visible spectrum of three samples of chitosan, chitosan with 1000 ppm $\mathrm{CaCl}_{2}$, and chitosan with 1000 ppm $\mathrm{MgSO}_{4}$. 


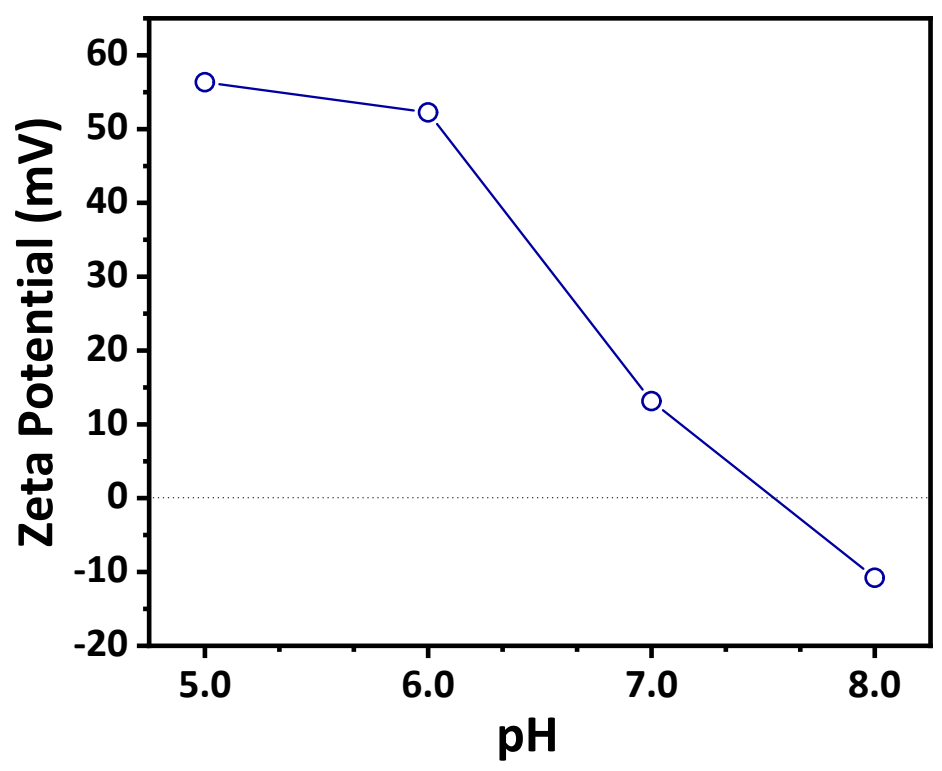

Figure S7. The Zeta potential of membrane 75-4t 

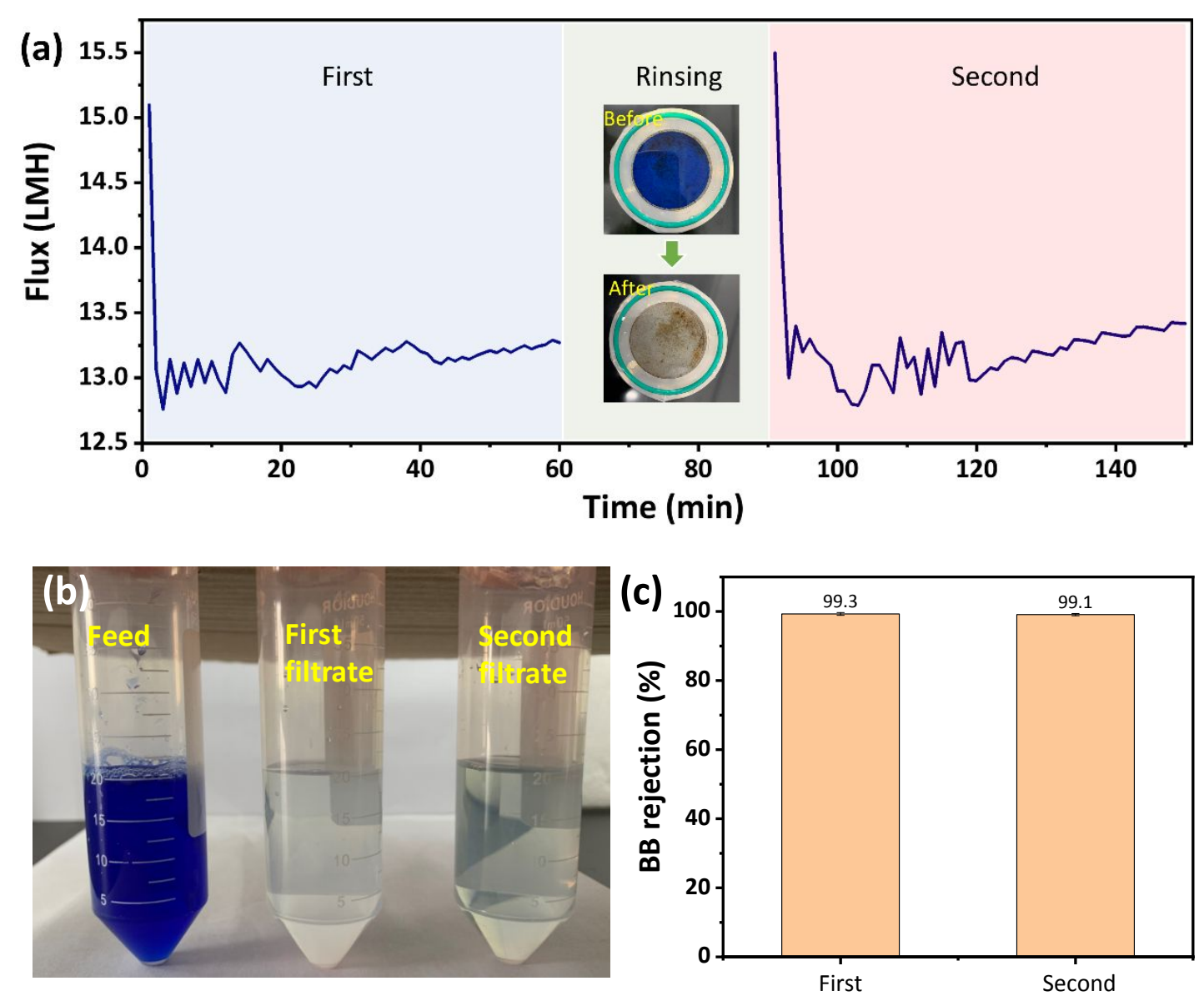

Figure S8. (a) The water flux and optical photograph of the membrane 75-4t before and after rinsing, (b) the feed and filtrate samples, and (c) BB dye rejection before and after solvent rinsing. 


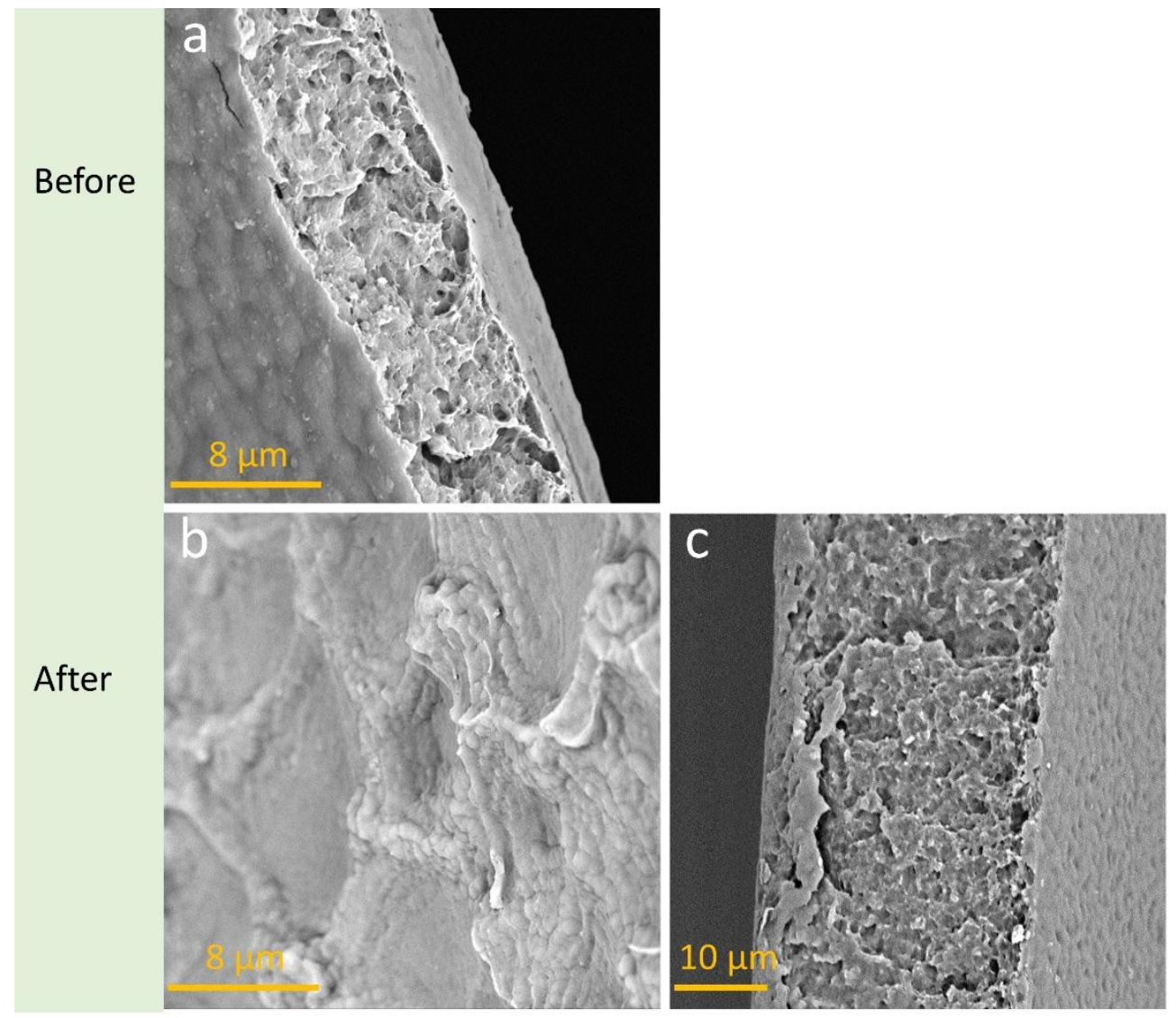

Figure S9. The SEM image of membrane 75-4t (a) the cross-section before regeneration, (b) the top surface after regeneration, and (c) the cross-section after regeneration. 